\title{
Aplicaciones computacionales de las ecuaciones diferenciales estocásticas
}

Recepción: Mayo de $2006 /$ Aceptación: Junio de 2006

(1) Eduardo Raffo Lecca

(2) Miguel Mejía Puente

\section{RESUMEN}

Los mètodos numèricos son herramientas efectivas para resolver los problemas de ingenierìa o ciencias, que utilizan ecuaciones diferenciales deterministicas. Asì tenemos los mètodos de Euler, Heun y los esquemas de Runge-Kutta. Estos algoritmos desafortunadamente no trabajan con ecuaciones diferenciales estocàsticas. La aplicación central se refiere a la utilización del cálculo estocástico en el campo financiero. El modelo de Black-Scholesy Merton para la opción del precio de los valores en mercados financieros, viene expresado mediante el movimiento browniano y las ecuaciones diferenciales estocásticas, proponiendo la valoración de los derivados financieros mediante el cálculo estocástico.

Palabras Clave: Cálculo estocástico, ecuaciones diferenciales estocásticas, procesos estocásticos.

Computational application OF THE STOCHASTIC DIFFERENTIAL EQUATIONS ABSTRACT

Numeric methods are effective tools to solve science or engineering problems, which use deterministic differential equations. We have Euler's and Heun's methods and RungeKutta's schemes. Unfortunately, these algorithms don't work with stochastic differential equations. The main application is referred to the utilization of stochastic calculus in the financial area. The Black-Scholes and Merton model of the price values option in the financial markets is expressed by the Brownian movement and the stochastic differential equation, proposing the financial derivatives valorization by means of the stochastic calculus.

Key words: Stochastic calculus, stochastic differential equations, stochastic processes.

\section{INTRODUCCIÓN}

El descubrimiento independiente del cálculo por Newton y Leibnitz, en el siglo XVIII, sentó las bases para el desarrollo de las matemáticas, ciencias y la ingeniería [1]. Uno de tales avances corresponde a la rama que las matemáticas denominan ecuaciones diferenciales.

Muchos problemas de matemáticas aplicadas, usan ecuaciones diferenciales ordinarias o ODE(Ordinary Differential Equation). En el caso más simple, una función diferencial $y=y(x)$ donde $x$ es una variable real, con derivada $y^{\prime}(x)$ satisface a una ecuación de la forma $y^{\prime}(x)=f(x, y(x))$ o simplemente:

$$
y^{\prime}=f(x, y)
$$

que es una ODE. Existe un valor inicial o IVP (Initial Value problem) en la solución y de (1.1) para los valores $\left(x_{0}, y_{0}\right)$, que satisfacen la condición inicial de la forma:

$$
y\left(x_{0}\right)=y_{0}
$$

para un infinito números de funciones diferentes y, que son solución de (1.1) [2].

Los ingenieros y científicos, frecuentemente hacen uso de las ecuaciones diferenciales para modelar los efectos del cambio, movimiento y crecimiento. Por ejemplo, ecuaciones diferenciales que predicen la dinámica poblacional, la estabilidad de la órbita en los satélites, o el movimiento de recursos en un mercado financiero [3].

Los modelos más precisos y sofisticados, con efectos aleatorios o estocásticos, vienen a ser más significativos. Siendo la aleatorización del sistema, la parte de mayor interés en el modelo. La solución de tales problemas, comprende una nueva área en matemáticas, denominada cálculo estocástico; y corresponde a una generalización estocástica del cálculo diferencial clásico.

Durante las dos décadas pasadas, hubo un acelerado interés en el desarrollo de métodos numéricos para las ecuaciones diferenciales estocásticas o SDE (Stochastic Differential Equations)[4]. Tal como se usa una ODE para modelar un sistema ideal sin ruido, se usa una SDE para modelar sistemas más realistas que incluyan el efecto del ruido.

Una SDE es la generalización de una ODE, cuando el ruido es perceptible 0 activo.

\footnotetext{
(1) Ingeniero Industrial. Profesor del Departamento de Ingeniería de Sistemas e Informática, UNMSM. E-mail: eraffol@unmsm.edu.pe

(2) Ingeniero Industrial. Profesor del Departamento de Ingeniería Industrial, PUCP

E-mail: mmejia@pucp.edu.pe
} 
En un problema de dinámica poblacional, el modelo determinista del crecimiento de la poblacional es la ecuación exponencial $\quad x^{\prime}=c x$, donde $c$ es el coeficiente de crecimiento maltusiano. Lo aleatorio del ambiente, puede ser modelado permitiendo a c, variar aleatoriamente como $c+\sigma \zeta_{t}$, para un proceso estocástico $\zeta_{t}$ con media cero; dando una SDE lineal de la forma:

$$
d X_{t}=c X_{t} d t+\sigma X_{t} d W_{t}
$$

\section{PROBABILIDADES Y PROCESOS ESTOCÁSTICOS}

Los conceptos de probabilidades y procesos estocásticos, necesarios para la comprensión de las SDE, son revisados a continuación.

\section{Probabilidades $\mathrm{y}$ eventos}

Cuando se lanza un dado, se observa como resultado una de las seis caras de los números $1,2, \ldots, 6$. Estos resultados denotados por $\omega_{1}, \omega_{2}, \Lambda, \omega_{6}$ respectivamente, generan el conjunto $\Omega=\left\{\omega_{1}, \omega_{2}, \Lambda, \omega_{6}\right\}$ denominado espacio muestral.

Si se lanza un dado un número de intentos y se contabiliza el número de veces $N_{i}$ que tiene el resultado $\omega_{i}$, se obtiene la frecuencia relativa $f_{i}=N_{i} / N$. Cuando $N$ es un número grande, se obtiene el

$$
\lim _{N \rightarrow \infty} f_{i}(N)=p_{i}
$$

conocido como la probabilidad del resultado $\omega_{i}$. Cumpliendo que $0 \leq p_{i} \leq 1$, para $i=1,2, \Lambda$, 6 y $\sum_{i=1}^{6} p_{i}=1$.

Cuando existe interés en un conjunto de resultados en el espacio muestral; $\operatorname{como} A=\left\{\omega_{1}, \omega_{3}, \omega_{5}\right\}$, el conjunto de las caras con números impares, se conoce como un evento. La probabilidad del evento denotada como $P(A)$, es calculada desde sus elementos, $P(A)=\sum_{\omega_{i} p_{i}}$. En particular el espacio muestral completo $\Omega$ es un evento.

La esencia de la información probabilística, puede ser sumarizada en la 3-tupla $(\Omega, \mathrm{A}, P)$, consistiendo de un espacio muestral $\Omega$, la colección de eventos A y la medida de la probabilidad $P$; siendo conocida técnicamente la colección de eventosA, como óálgebra.

\section{Variables aleatorias}

Una variable aleatoria es una función que asocia un número con cada punto en el espacio muestral del experimento. En las aplicaciones de las probabilidades, existe el interés en las funciones de los resultados, antes que los resultados.

El número $X(\omega)$, provee información acerca del experimento en el espacio de probabilidad $(\Omega, \mathrm{A}, P)$; ello corresponde a los valores que toma alguna función $X: \Omega \rightarrow \mathfrak{R}$, la cual se denomina variable aleatoria.

De una manera mas genérica, los valores de $X$ se encuentran en el conjunto $E$. Los más usuales ejemplos de $E$, son el conjunto de todos los enteros no negativos, el conjunto de todos los enteros y el conjunto de todos los números reales. Cuando $E$ es finito o contablemente infinito, se dice que $X$ es una variable discreta.

Sea el espacio de probabilidad $(\Omega, \mathrm{A}, P)$; se dice que una función $X: \Omega \rightarrow \Re$ es una variable aleatoria, si:

$$
\{\omega \in \Omega: X(\omega) \leq a\} \in \text { A para cada } a \in \mathfrak{R}(2.1)
$$

En un experimento de observar un vehículo durante un determinado tiempo $\mathrm{T}$, se tienen las siguientes variables aleatorias

$$
\begin{aligned}
& X_{t}(\omega)=\omega(t), \\
& Y_{t}(\omega)=\int_{0}^{t} \omega(t) d s, \\
& Z_{t}(\omega)=\int_{0}^{t} Y_{u}(\omega) d u=\int_{0}^{t} \int_{0}^{u} \omega(s) d s d u
\end{aligned}
$$

Se observa que para $t \in[0, T]$, y cada $\omega \in \Omega$, las variables $X_{t}, Y_{t}$ y $Z_{t}$ son variables aleatorias en $\Omega$; correspondiendo a la aceleración, la velocidad y la posición, respectivamente.

\section{Proceso estocástico}

Un proceso estocástico, es una colección parametrizada de variables aleatorias

$$
\left\{X_{t}\right\}_{t \in I}
$$

definidas en un espacio de $\operatorname{probabilidad}(\Omega, \mathrm{A}, P)$, indexados por un parámetro $t \in I \subset \Re$, que a menudo se le interpreta como tiempo. Para cada $t$ fijo, se tiene una variable aleatoria.

$$
\omega \rightarrow X_{t}(\omega), \omega \in \Omega
$$

y para cada $\omega \in \Omega$, se tiene una trayectoria

$$
t \rightarrow X_{t}(\omega), t \in I
$$

El conjunto tiempo está en $I$ y asume que es común al espacio de probabilidad $(\Omega, \mathrm{A}, P)$. Un proceso estocástico $X=\{X(t), t \in I\}$ es una función de dos variables $X: I x \Omega \rightarrow \Re \quad$, donde $X(t)=X(t$,$) es$ una variable aleatoria por cada $t \in I$. Por cada $\omega \in \Omega$, se denomina $X(., \omega): I \rightarrow \Re$ una realización o una trayectoria (sample path). 
La variable de velocidad $Y_{t}$, es la velocidad del vehículo en el tiempo $t$, y la colección $\left\{Y_{t} ; 0 \leq t \leq T\right\}$ es un proceso estocástico en tiempo continuo con el estado del espacio $\mathfrak{R}_{+}=[0, \infty)$.

La teoría de los procesos markovianos, fue desarrollada sobre el supuesto que el futuro, está condicionado por el conocimiento del valor presente. Correspondiendo a uno de los procesos estándares en el estudio de los procesos estocásticos.

Un proceso estocástico es llamado una cadena de Markov, cuando:

$$
P\left\{X_{n+1}=j \mid X_{0}, \Lambda, X_{n}\right\}=P\left\{X_{n+1}=j \mid X_{n}\right\}
$$

para todo $j \in E \mathrm{y} n \in \mathrm{N}$.

Existe una clase de procesos estocásticos, con incrementos independientes, esto es que las variables aleatorias $X\left(t_{j+1}\right)-X\left(t_{j}\right), j=0,1, \Lambda, n-1$ son independientes para cualquier combinación finita de instantes tiempos $t_{0}<t_{1}<\Lambda<t_{n}$ en $T$. Sea $t_{0}$ el más pequeño instante tiempo en $T$, entonces las variables aleatorias $X\left(t_{0}\right)$ y $X\left(t_{j}\right)-X\left(t_{0}\right)$ para cualquier $t_{j}$ en $T$, también requieren ser independientes.

$X$ tiene incrementos independientes, si los incrementos $d X(s)$ y $d X(t)$ sobre los intervalos $[s, s+d s]$ y $[t, t+d t]$ son independientes. Si cada incremento tiene una distribución normal, se dice que $X$ tiene una distribución normal o proceso Gaussiano.

Los procesos Wiener son un ejemplo de procesos con incrementos independientes, $W=\{W(t), t \geq 0\}$, esto es un proceso Gaussiano con incrementos independientes, con

$$
\begin{aligned}
& W(0)=0, E(W(t))=0, \operatorname{Var}(W(t)-W(s))=t-s, \\
& W(t)-W(s) \sim N(0, \sqrt{t-s})
\end{aligned}
$$

para todo $0 \leq s \leq t$.

Este proceso estocástico, fue propuesto por Wiener como una descripción matemática del movimiento Browniano.

En 1827 el botánico inglés Robert Brown, analizó el movimiento de partículas de polen en el agua, y lo relacionó con las teorías vitalistas de la vida, argumentando que ese movimiento era propio de la materia viviente, y ligado con los mecanismos de la reproducción. Sin embargo, en sus trabajos finales, concluye que ese movimiento errático era de naturaleza mecánica y no dependía del carácter orgánico ni inorgánico de los objetos considerados.

En 1905, Albert Einstein construyó un modelo mate- mático para explicar el fenómeno, y lo denominó "movimiento Browniano" en honor a su descubridor.

\section{Generadores de números aleatorios}

El término aleatorio, viene de una característica que se atribuye a cierta clase de procesos; en el que no se puede reconocer ningún orden, por lo cual no es posible predecir su estado.

Los números aleatorios, son números de una sucesión que cumplen con ser:

- Uniformemente distribuidos

- Ser aleatorios en su aparición

Estos números son útiles en una gran variedad de aplicaciones:

1. Programación de computadoras

2. Análisis Numérico

3. Muestreo

4. Simulación

5. Teoría de decisiones

6. Juegos de computadoras

La historia de los números aleatorios, en el trabajo científico se remonta a los bolos, ruletas y cartas. En 1927, L.C. Tippett había conseguido una tabla de cerca de 40,000 números aleatorios; en 1939, la Rand Corporation produjo una tabla de un millón de números aleatorios. Con la llegada de la computadora, se pensó almacenar estas tablas usando su memoria. Al no ser práctica esta idea, fueron reemplazados por las operaciones aritméticas [5].

Von Neumann y Metrópolis (1946), fueron los primeros en proponer el uso de las operaciones aritméticas en una computadora, creando el método del cuadrado central o medio.

Siendo cada número aleatorio, generado completamente por procesos determinísticos desde su predecesor. La secuencia generada de esta manera, es denominada seudo aleatoria, cuasi aleatoria o seudo casual.

Un generador de números aleatorios debe cumplir con las siguientes propiedades:

- Ser breve en el uso de la memoria.

- Tener periodo largo.

- Ser rápido en ejecución.

Sea un entero $x_{n}$, no negativo y menor que $m$, la fracción:

$$
U_{n}=x_{n} / m
$$

se encuentra entre cero y uno. 
Lehmer (1948), presentó la formula para generar la secuencia $\left\{x_{n}\right\}$

$$
x_{n+1} \equiv a x_{n}+c \quad(\bmod m)
$$

conocida como secuencia congruencial lineal; siendo:

$$
\begin{aligned}
& x_{0}=\text { la semilla } \\
& a=\text { el multiplicador, } a \geq 0 \\
& c=\text { el incremento, } c \geq 0 \\
& m=\text { el módulo } m \geq a, m>c, m>x_{0}
\end{aligned}
$$

Por ejemplo la secuencia obtenida con $x_{0}=a=c=4$ y $m=10$, es $0,4,0,4,0,4,0,4$. Los modernos lenguajes de programación, hacen uso de la teoría de Lehmer para implementar funciones que generan números seudo aleatorios; como sucede con la función rand en MATLAB.

Considere la situación de lanzar una moneda, si cae cara vale 1 , en otro caso -1 :

$$
X_{n}= \begin{cases}1, & \text { cara } \\ -1, & \text { en otro caso }\end{cases}
$$

Este proceso es conocido como random walk(caminata al azar), tiene $E\left(X_{n}\right)=0$ y $\operatorname{Var}\left(X_{n}\right)=1$. En la figura 1, se presenta una simulación haciendo uso de los números aleatorios y en la figura 2 , el código MATLAB respectivo.

\section{CÁLCULO ESTOCÁSTICO}

Un proceso estocástico es denominado Wiener, con coeficientes drift $\mu$ y diffusion $\sigma$, si posee la siguiente conducta dinámica:

$$
\begin{aligned}
& d X=\mu d t+\sigma d W, \\
& X(0)=x_{0}
\end{aligned}
$$

donde $x_{0}, \mu \mathrm{y} \sigma$ son constantes.

Desde el intervalo $[0, t]$, la expresión (3.1), tiene por solución:

$$
X(t)=x_{0}+\mu t+\sigma W(t)
$$

La distribución de $X$, viene dada por $N\left(x_{0}+\mu t, \sigma \sqrt{t}\right)$.

Sea la conducta dinámica:

$$
\begin{aligned}
& d X=\mu\left(t, X_{t}\right) d t+\sigma\left(t, X_{t}\right) d W \quad \mathrm{y} \\
& X(0)=x_{0}
\end{aligned}
$$

con una velocidad determinística $\mu\left(t, X_{t}\right)$ y una distribución Gaussiana, amplificada por $\sigma\left(t, X_{t}\right)$. Al dividirse por $d t$, se obtiene la ODE estocástica.

$$
\begin{aligned}
& \frac{d X}{d t}=\mu\left(t, X_{t}\right)+\sigma\left(t, X_{t}\right) v_{t}, \\
& v_{t}=\frac{d W}{d t}
\end{aligned}
$$

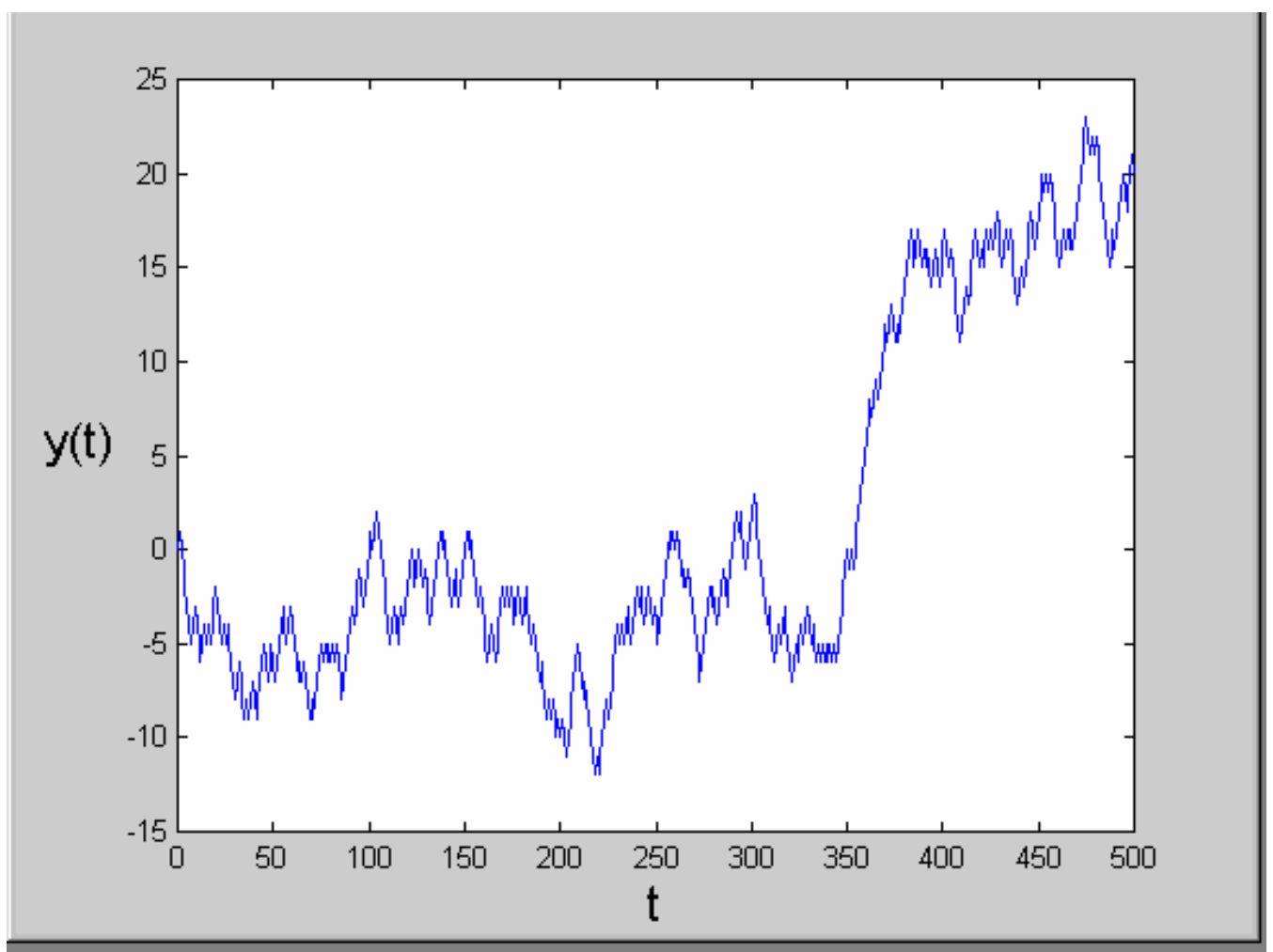

Figura 1. Gráfica de la caminata al azar 
function SimCoin()

$\%$ Simulation del proceso random walk

$\%$ E. Raffo Lecca

$\%$

$\%$ Datos

$\% \mathrm{dt}=$ longitud del segmento

$\% \mathrm{t}=$ es el vector tiempo

$\% \mathrm{~T}=$ numero de segmentos

$\%$ Resultados

$\%$ y $=$ es el vector de aproximacion

$\mathrm{dt}=1$;

rand('state',100);

$\mathrm{T}=500$;

$\mathrm{y}=\mathrm{zeros}(\mathrm{T}+1,1)$;

$\mathrm{t}=0$ :dt:T;

for $\mathrm{i}=1: \mathrm{T}$

if rand $<0.5$

$\mathrm{x}=1$;

else

$\mathrm{x}=-1$;

end

$y(i+1)=y(i)+x$;

end

$\mathrm{U}=$ mean $(\mathrm{y})$

plot(t,y,'b-')

xlabel('t','Fontsize',16)

ylabel('y(t),'Fontsize',16,'Rotation',0,'HorizontalAlignment','right')

Figura 2. M-file simCoin

Que es la forma de presentar el proceso $W$ denominado Wiener.

Esta ecuación en la forma integral, se escribe como

$$
X_{t}=x_{0}+\int_{0}^{t} \mu\left(s, X_{s}\right) d s+\int_{0}^{t} \sigma\left(s, X_{s}\right) d W_{s}
$$

que se interpreta como que la primera integral, es la de Riemann para cada trayectoria $X$ y la segunda integral, una integral estocástica que se basa en la teoría del cálculo Ito.

Las SDE lineales y de ruido multiplicativo, con coeficientes constantes homogéneos, o de la forma

$$
d X_{t}=a X_{t} d t+b X_{t} d W_{t}
$$

tienen como solución explícita

$$
X_{t}=X_{0} \exp \left(\left(a-\frac{1}{2} b^{2}\right) t+b W_{t}\right)
$$

para $t \in[0, T]$, con un proceso Wiener $W=\{W(t), t \geq 0\}$.

Desde la ecuación (3.6), se determinan los valores de la solución exacta para el sample path del proceso Wiener, usando:

$$
X_{\tau_{n}}=X_{0} \exp \left(\left(a-\frac{1}{2} b^{2}\right) \tau_{n}+b \sum_{i=1}^{n} \Delta W_{i-1}\right)
$$

\section{MÉTODOS NUMÉRICOS ESTOCÁSTICOS}

Los métodos numéricos, son las herramientas efectivas para resolver los problemas de ingeniería o de ciencias, que utilizan ecuaciones diferenciales deterministas. Allí se encuentran los métodos de Euler, Heun y los esquemas de Runge-Kutta. Estos algoritmos desafortunadamente no trabajan con SDE [6].

Un proceso Ito, $X=\left\{X_{t}, t \geq 0\right\}$ tiene la forma

$$
X_{t}=X_{0}+\int_{0}^{t} a\left(X_{s}\right) d s+\int_{0}^{t} b\left(X_{s}\right) d W s
$$

para $t \geq 0$; con IVP igual a $X_{0}=x_{0}$. Se observa una componente continua de variación lenta, llamada drift, y una componente aleatoria continua de variación rápida, llamada diffusion.

La segunda integral en (4.1) es una integral estocástica Ito, con respecto al proceso Wiener $W=\left\{W_{t}, t \geq 0\right\}$.

La ecuación integral en (4.1), es a menudo escrita por su forma diferencial

$$
d X_{t}=a\left(X_{t}\right)+b\left(X_{t}\right) d W_{t}
$$

conocida como la SDE Ito.

Se considera una discretización del tiempo $(\tau)_{j}$ con:

$$
0=\tau_{0}<\tau_{1}<\Lambda \tau_{n}<\Lambda<\tau_{N}=T
$$

en el intervalo $[0, T]$, siendo el caso más simple el equidistante, donde el tamaño del paso corresponde a:

$$
\delta=\frac{T}{N}
$$

\section{Aproximación de Euler}

La más simple aproximación heurística en tiempo discreto es la generalización estocástica de la aproximación de Euler, a menudo denominada aproximación de Euler-Maruyama.

Para el SDE de (4.2), el proceso $X_{t}$ se aproxima por

$$
Y_{n+1}=Y_{n}+a\left(Y_{n}\right) \Delta_{n}+b\left(Y_{n}\right) \Delta W_{n}, \quad n=0,1, \Lambda, N-1(4.5
$$

con IVP

$$
Y_{0}=x_{0}
$$

donde

$$
\Delta_{n}=\tau_{n+1}-\tau_{n}=\delta
$$


y

$$
\Delta W_{n}=W_{\tau_{n+1}}-W_{t_{n}}
$$

para $n=0,1, \Lambda, N-1$.

Las variables aleatorias $\Delta W_{n}$ definidas en (4.8), son variables aleatorias independientes distribuidas normalmente, con media y varianza

$$
E\left(\Delta W_{n}\right)=0 \quad y \quad E\left(\left(\Delta W_{n}\right)^{2}\right)=\Delta_{n}
$$

La generación de las variables aleatorias $\Delta W_{n}$, se consigue usando la transformación desde (4.9),

$$
\Delta W_{n}=\zeta \Delta_{n}^{1 / 2}
$$

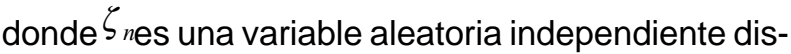
tribuida en forma normal en $N(0,1)$.

Para realizar simulaciones, se provee de generadores de números y procesos aleatorios; tal como la función randn de MATLAB, que genera variables normalmente distribuidas en $N(0,1)$ :

\section{$d w=\operatorname{sqrt}(d t)^{\star} \operatorname{randn}(1, n)$;}

Para el efecto de estimar los procesos de Ito, mediante la simulación, se hace necesario que la aproximación de las trayectorias o el camino de muestras (sample path) sea cerrada a este proceso.
Sean las funciones drift y difusion, siguientes:

$$
a\left(X_{t}\right)=1.5 X_{t} \quad y \quad b\left(X_{t}\right)=1.0 X_{t}
$$

Invocando a la función SdeEulerDemo.m, se consigue la gráfica de la figura 3 , que presenta la aproximación del esquema de Euler y la solución real. El M-file SdeEulerMaruyama, aparece en la figura 4.

La aplicación del demo, se presenta en el M-file SdeEulerDemo, que aparece en la figura 5, y es el que efectúa la llamada al esquema Euler-Maruyama.

Se considera el error absoluto al final del tiempo, en el instante $T$ :

$$
\in(\delta)=E\left(\left|X_{T}-Y_{N}\right|\right)
$$

El proceso de aproximación $Y$, converge en un sentido fuerte con orden $\gamma \in(0, \infty)$ si existe una constante finita $k$ y una constante $\delta_{0}$, con $k>0$ y $\delta_{0}>0$, tal que

$$
E\left(\left|X_{T}-Y_{N}\right|\right) \leq k \delta^{\gamma}
$$

para cualquier tiempo de discretización, con paso máximo $\delta \in\left(0, \delta_{0}\right)$.

Como los incrementos $\Delta W_{n}$ del proceso Wiener son $\delta^{1 / 2}$ y no $\delta$, entonces la aproximación de EulerMaruyama posee un orden fuerte de $\gamma=0.5$.

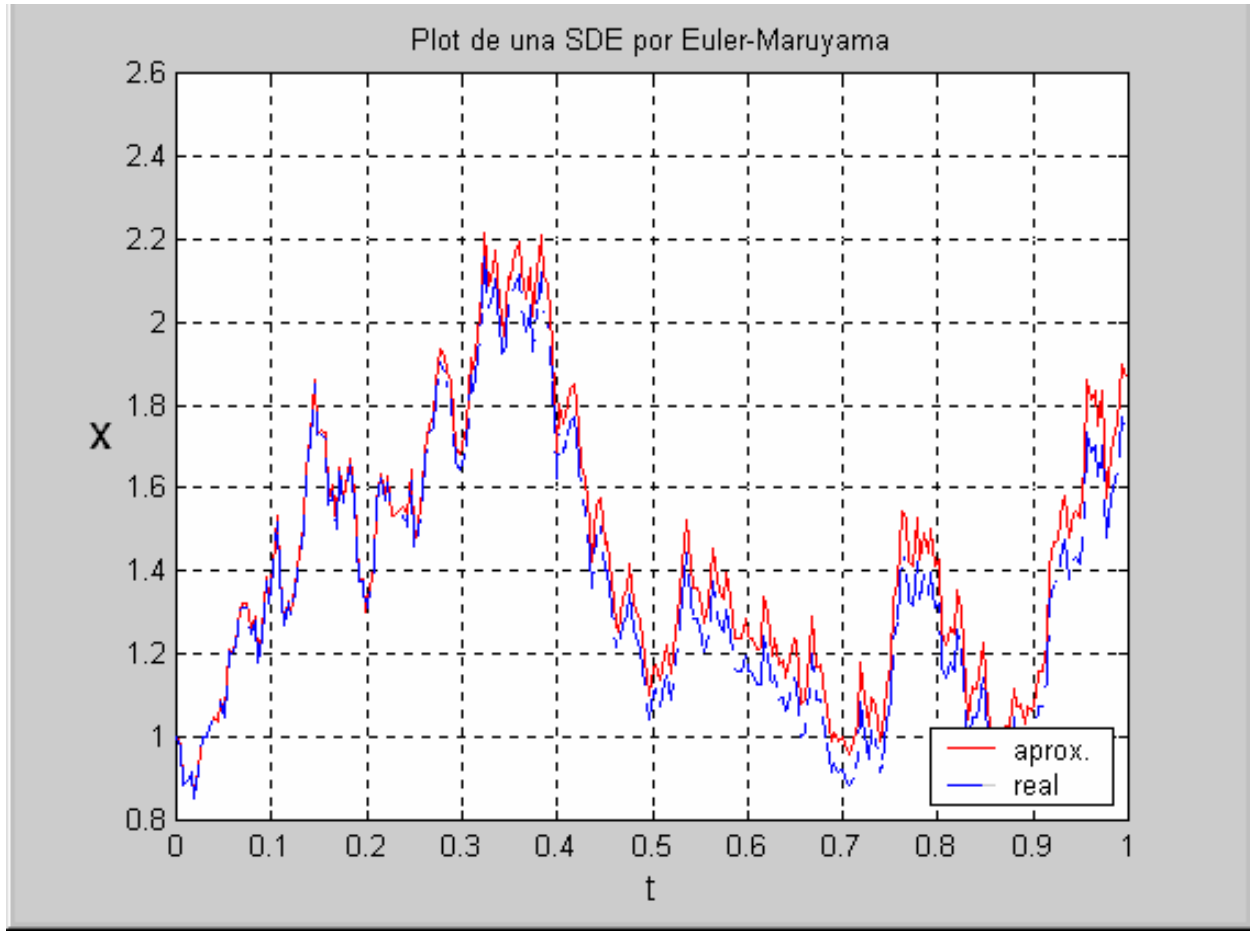

Figura 3. Gráfica del esquema Euler 


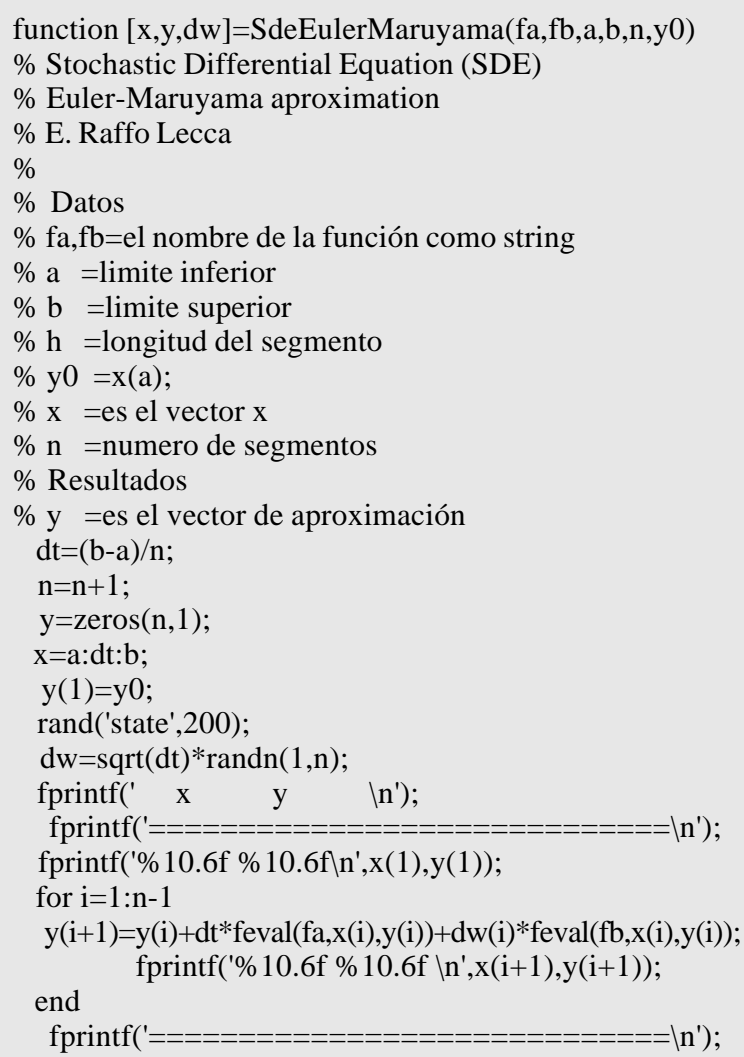

\section{Esquema Milstein}

La representación del proceso Ito en la fórmula estocástica de Taylor, para una determinada simplificación corresponde a

$X_{t}=X_{t_{0}}+a\left(X_{t_{0}}\right) \int_{t_{0}}^{t} d s+b\left(X_{t_{0}}\right) \int_{t_{0}}^{t} d W_{s}+b\left(X_{t_{0}}\right) b^{\prime}\left(X_{t_{0}}\right) \int_{t_{0}}^{t} \int_{t_{0}}^{s_{2}} d W_{1} d W_{2}+R$

La doble integral de Wiener en (4.12), es calculada como

$$
\int_{\tau_{n}}^{\tau_{n+1}} \int_{\tau_{n}}^{s_{2}} d W_{s_{1}} d W_{s_{2}}=\frac{1}{2}\left\{\left(\Delta W_{n}\right)^{2}-\Delta_{n}\right\}
$$

Desde la aproximación de Euler-Maruyama en (4.5), e incluyendo el resultado de (4.13) en (4.12) se obtiene el esquema de Milstein

$$
Y_{n+1}=Y_{n}+a \Delta_{n}+b \Delta W_{n}+\frac{b b^{\prime}}{2}\left\{\left(\Delta W_{n}\right)^{2}-\Delta_{n}\right\}
$$

donde Milstein converge con un orden fuerte de $\gamma=1$ desde (4.11)

$$
E\left(\left|X_{T}-Y_{N}\right|\right) \leq k \delta
$$

Si $b\left(X_{t}\right)$ no depende de $X_{t}$, entonces Euler-Maruyama y el esquema Milstein coinciden; porque $b^{\prime}=\partial b / \partial X_{t}=0$.

Invocando a la función SdeMilsteinDemo.m, se consigue la gráfica de la figura 6, que presenta la

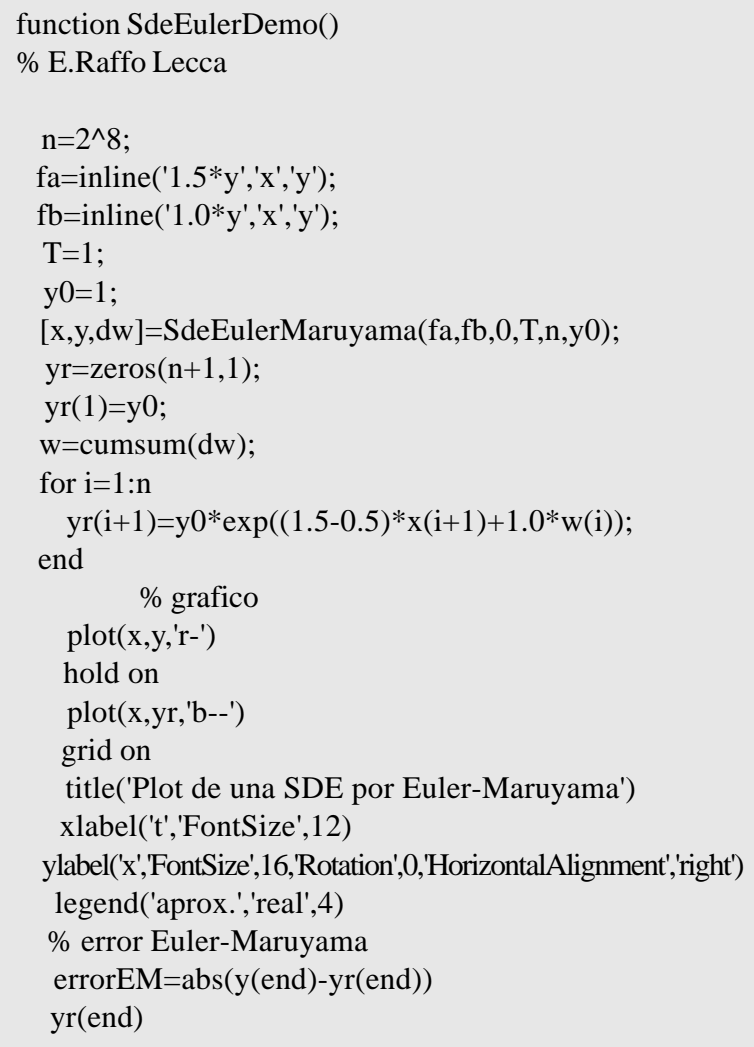

\section{Figura 5. M-file SdeEulerDemo}

aproximación del esquema de Milstein y la solución real. El M-file SdeMilstein, aparece en la figura 7 .

La aplicación del demo, se presenta en el M-file SdeMilsteinDemo, que aparece en la figura 8, y es el que efectúa la llamada al esquema Euler-Maruyama.

Efectuando 1000 sample path, y calculando el promedio para cada una de las realizaciones, se ha conseguido la gráfica que se presenta en la figura 9 . El esquema utilizado en esta simulación corresponde al Euler-Maruyama. En la figura 10, se presenta el M-file para esta simulación, el simEuler.m.

\section{APIICACIÓN}

Los modelos y técnicas utilizados hoy en día por los analistas financieros, están basados en el trabajo "The Pricing of Options and Corporaties Liabilities", ganador al premio Nóbel de 1997 en Ciencias Económicas, desarrollado por Black-Scholes y R. Merton (BSM).

La introducción de los derivados financieros, tales como las opciones y futuros sobre subyacentes (tí- 


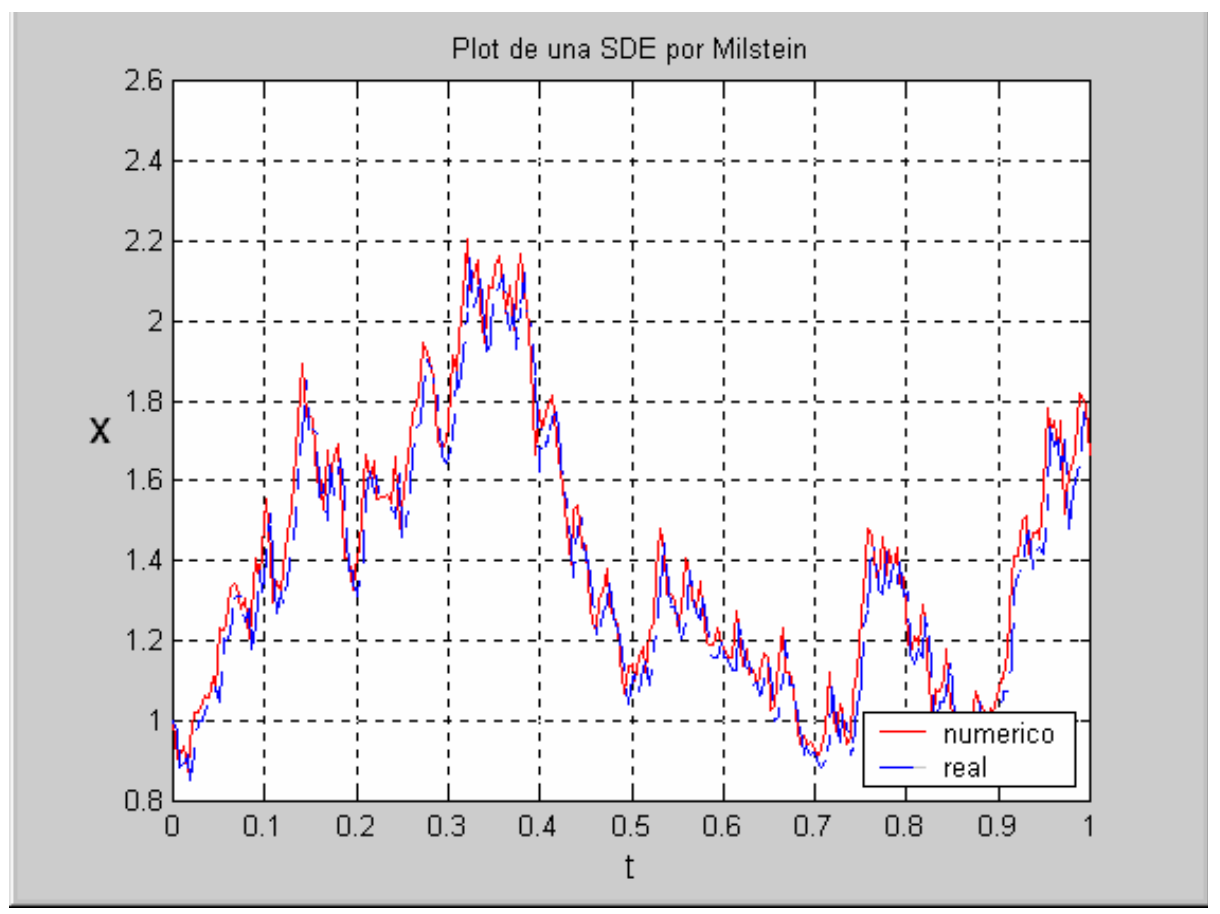

Figura 6. Gráfica del esquema Milstein

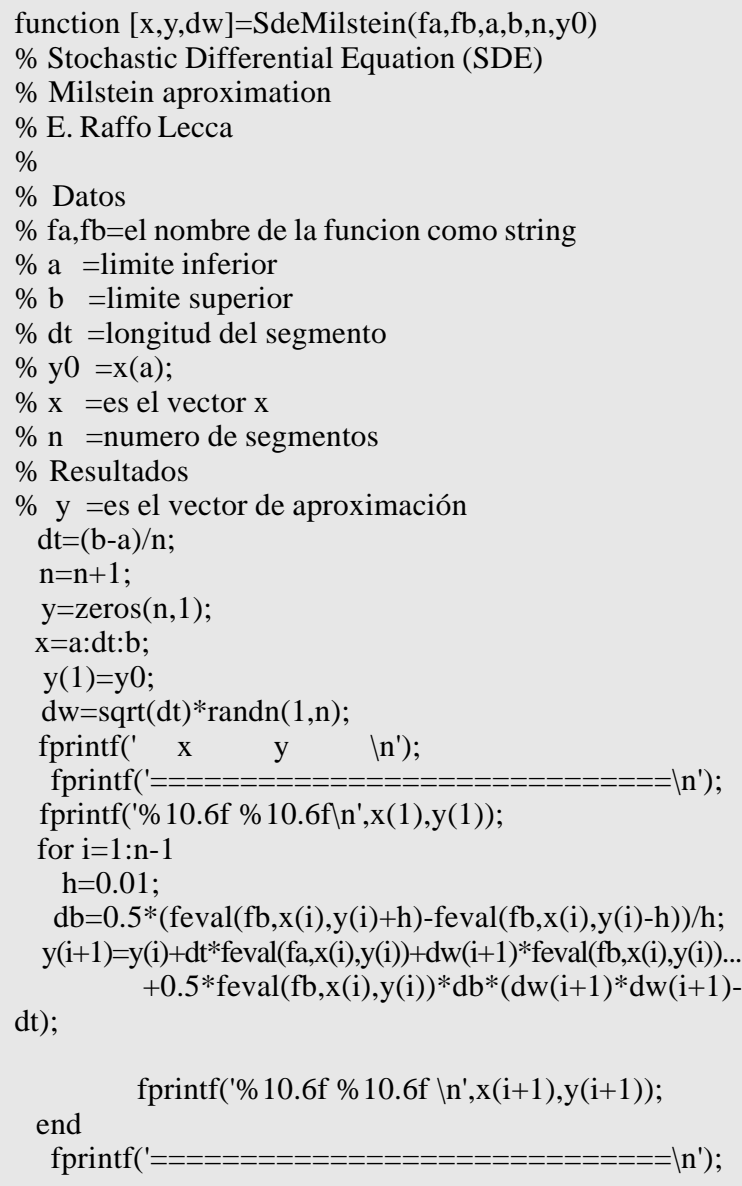

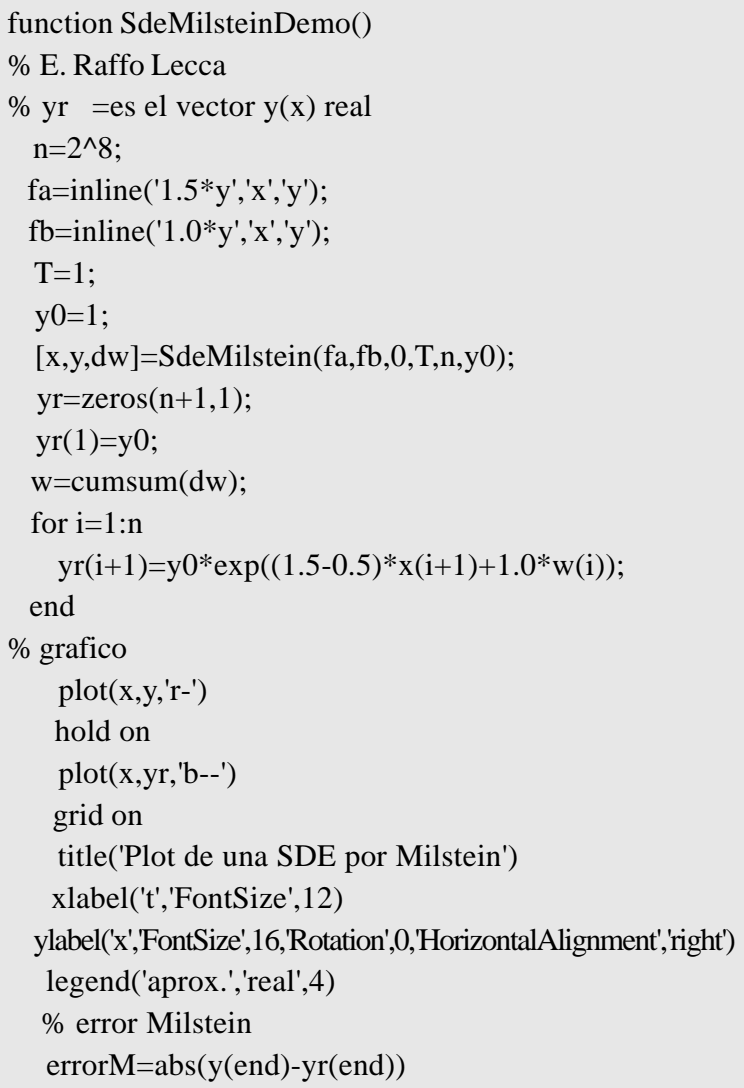

Figura 8. SdeMilsteinDemo 


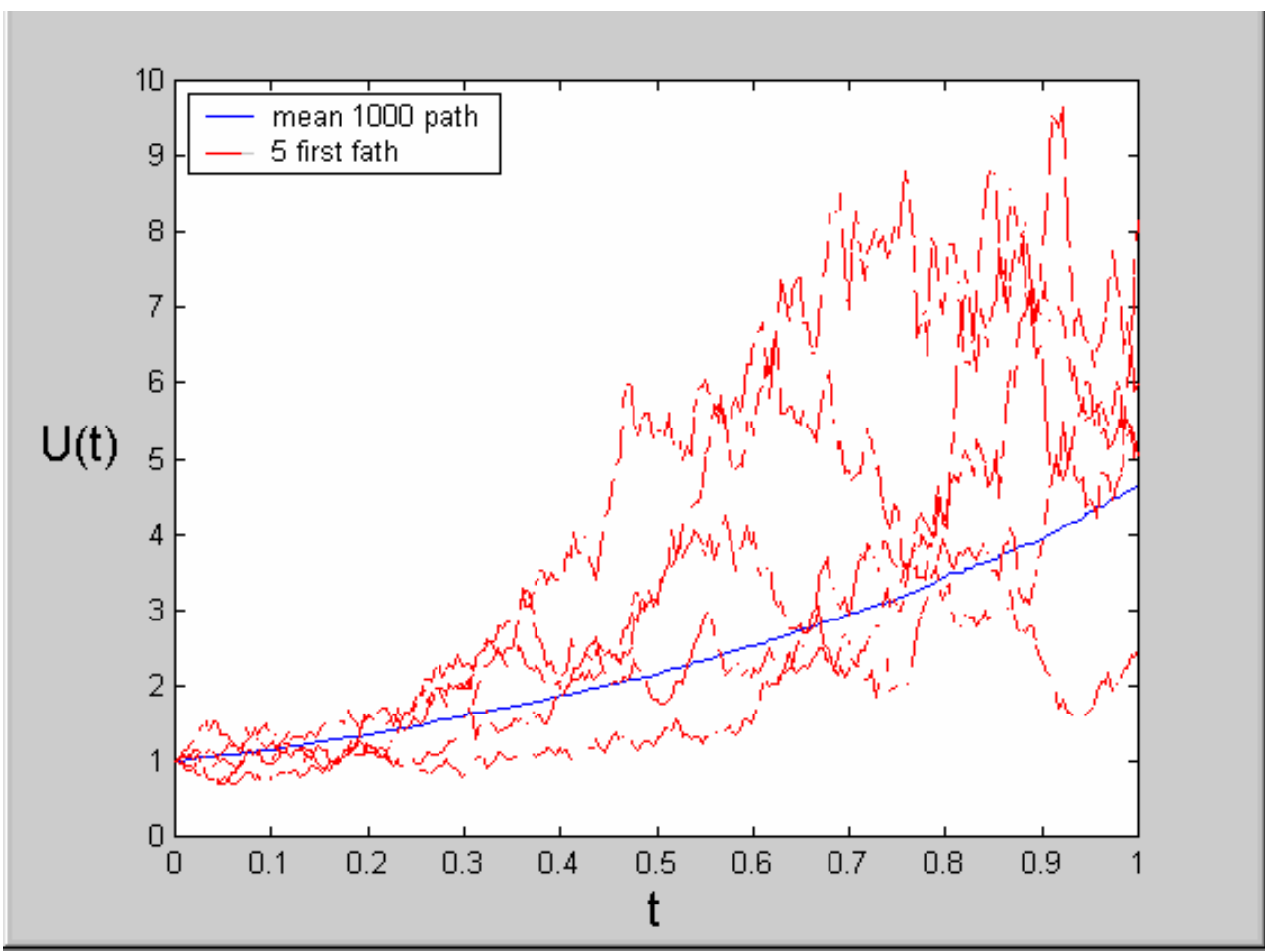

Figura 9. Gráfica del esquema Milstein

function SimEuler()

$\%$ Stochastic Differential Equation (SDE)

$\%$ Simulation Euler-Maruyama aproximation

\section{$\%$ E. Raffo Lecca}

$\%$

\section{$\%$ Datos}

$\%$ lambda $=$ parametro de $\mathrm{a}(\mathrm{X})$

$\% \mathrm{mu}=$ parametro de $\mathrm{b}(\mathrm{X})$

$\% \mathrm{dX}=$ lambda $* \mathrm{Xdt}+\mathrm{mu} * \mathrm{X} * \mathrm{dw}$

$\% \mathrm{dt}=$ longitud del segmento

$\% \mathrm{y} 0=\mathrm{X}$ zero

$\% \mathrm{t}=$ es el vector tiempo

$\% \mathrm{~N}=$ numero de segmentos

$\% \mathrm{M}=$ numero de corridas

$\%$ Resultados

$\% \mathrm{y}=$ es el vector de aproximacion a $\mathrm{X}$

$\mathrm{N}=2^{\wedge} 8 ; \mathrm{M}=1000$;

$\mathrm{dt}=1 / \mathrm{N}$;

rand('state',100);
$\mathrm{T}=1$;

lambda $=1.5 ; \mathrm{mu}=1 ; \mathrm{y} 0=1$;

$\mathrm{y}=\mathrm{zeros}(\mathrm{M}, \mathrm{N}+1)$;

$\mathrm{t}=0: \mathrm{dt}: \mathrm{T}$

$\mathrm{dw}=\operatorname{sqrt}(\mathrm{dt}) * \operatorname{randn}(\mathrm{M}, \mathrm{N})$;

yant=y0*ones $(\mathrm{M}, 1)$

$\mathrm{y}(:, 1)=$ yant $(:)$;

for $\mathrm{i}=1: \mathrm{N}$

$\mathrm{y}(:, \mathrm{i}+1)=y a n t(:)+$ lambda*dt*yant(:)+mu*yant(:).*dw(:,i); $\operatorname{yant}(:)=y(:, i+1)$;

end

ymean $=$ mean $(\mathrm{y})$;

plot(t,ymean,'b-'), hold on

plot(t,y(1:5,:),'r--')

xlabel('t','Fontsize',16)

ylabel('U(t),'Fontsize',16,'Rotation',0,'HorizontalAlignment','right')

legend('mean 1000 path','5 first path',2)

hold off 
tulos, obligaciones, moneda extranjera, etc.), han generado una nueva temática en la seguridad de los riesgos financieros. Un derivado financiero, consiste en comprar un seguro que cubra los activos arriesgados en el mercado (coberturista). Por su parte un operador especulador, la utilizará para apostar en torno al futuro del mercado y tratar de obtener mayor rentabilidad [7].

El modelo de la opción call da a su comprador el derecho, pero no la obligación a comprar un activo subyacente a un precio predeterminado en una fecha concreta. El vendedor de la opción call tiene la obligación de vender el activo en el caso de que el comprador ejerza el derecho a comprar. El objetivo del comprador es hacer dinero si el valor del stock cubre el precio de la opción [8].

El modelo de Black-Scholes o la fórmula de valoración de opciones (publicado en el Journal of Political Economy de mayo / junio de 1973), proporciona el valor de una opción de compra (call) "europea", la que no se puede ejecutar mas que en la fecha de vencimiento; sobre el precio de una acción de un determinado título $P(t)$, con precio de ejercicio $K$ (strike price).

Sea $P(t)$, para denotar el precio de un stock en el tiempo $t \geq 0$. Un modelo estándar asume que $\frac{d P}{P}$, el cambio relativo del precio viene de acuerdo a la SDE.

$$
\frac{d P}{P}=\mu d t+\sigma d W
$$

para ciertas constantes $\mu$ y $\sigma$, denominadas rumbo o tanto de rendimiento medio y volatilidad o variabilidad del precio, respectivamente (drifty diffusion). En otras palabras se supone que el cambio sigue un movimiento Browniano, de la forma

$$
\begin{aligned}
& d P=\mu P d t+\sigma P d W \\
& P(0)=p_{0}
\end{aligned}
$$

Se observa que el beneficio en el momento del vencimiento, viene dado por $h\left(P_{T}\right)=\left(P_{T}-K\right)_{+}=\max \left\{0, P_{T}-K\right\}$; es decir que el comprador obtiene beneficio, solamente si el precio de la acción al vencimiento es superior al precio del ejercicio mas la prima.

El estudio de los modelos de precios haciendo uso del movimiento Browniano, no es reciente; ya en 1900, Louis Bachelier en su "Théorie de la spéculation", propuso un modelo para precios especulativos; $y$ en 1960, el economista Paul Anthony Samuelson (Premio Nóbel de Economía en 1970, por el trabajo científico de desarrollar la teoría económica estática y dinámica y haber contribuido activamente a elevar el nivel del análisis en la ciencia económica), utilizó el movimiento Browniano geométrico(propagación de la exponencial del movimiento Browniano) para modelar los precios sujetos a incertidumbres.

En los trabajos de Merton y Black-Scholes, se asocia el movimiento Browniano geométrico al movimiento de los precios; y se liga con la teoría del cálculo estocástico o de lto, desarrollada por el matemático japonés Kiyosi Ito en los años de 1940.

El fin de un derivado financiero es el de minimizar riesgos para el coberturista; por su parte el modelo BSM permite tanto al comprador como al vendedor de un derivado, compensar los riesgos futuros producidos por la incertidumbre del movimiento de los precios.

En las figuras 11,12 y 13 , se presentan: el sample path resultante del modelo BSM, el programa de simulación y el histograma del precio al final del periodo, respectivamente. Se hace uso del esquema Milstein.

Los datos usados para la corrida anterior han sido tomados desde[9], y corresponden a:

$\sigma=53.5265 \%{ }^{\prime} P_{0}=\$ 94.00$ y $T=1$ año $\mu=0.053541$

Las compañías usan opciones y otros instrumentos derivados para reducir sus propios riesgos. Los bancos y otras instituciones financieras apelan al modelo BSM para desarrollar y determinar el valor de nuevos productos, o vender soluciones financieras a la medida a sus clientes. Como también para reducir los riesgos que surgen de su actuación en los mercados financieros y físicos.

\section{CONCIUSIONES}

Durante las dos décadas pasadas, hubo un acelerado interés en el desarrollo de métodos numéricos para las ecuaciones diferenciales estocásticas o SDE. Así como se usa una ODE (ecuación diferencial ordinaria) para modelar un sistema ideal sin ruido, se usa SDE para modelar sistemas más realistas que incluyan el efecto del ruido.

El cálculo Ito es conocido y utilizado por físicos e ingenieros; siendo por mucho tiempo desconocido fuera de estos grupos de profesionales; pero la aplicación al campo de las finanzas, ha ocasionado hacer populares las reglas del cálculo lto.

A la pregunta ¿hasta cuánto estaría dispuesto a pagar por una opción call ?, es lo que se plantea en el modelo de BSM, para cuando se está interesado en una opción call Europea que le permita compensar los riesgos futuros, producidos por la incertidumbre del movimiento de los precios. 


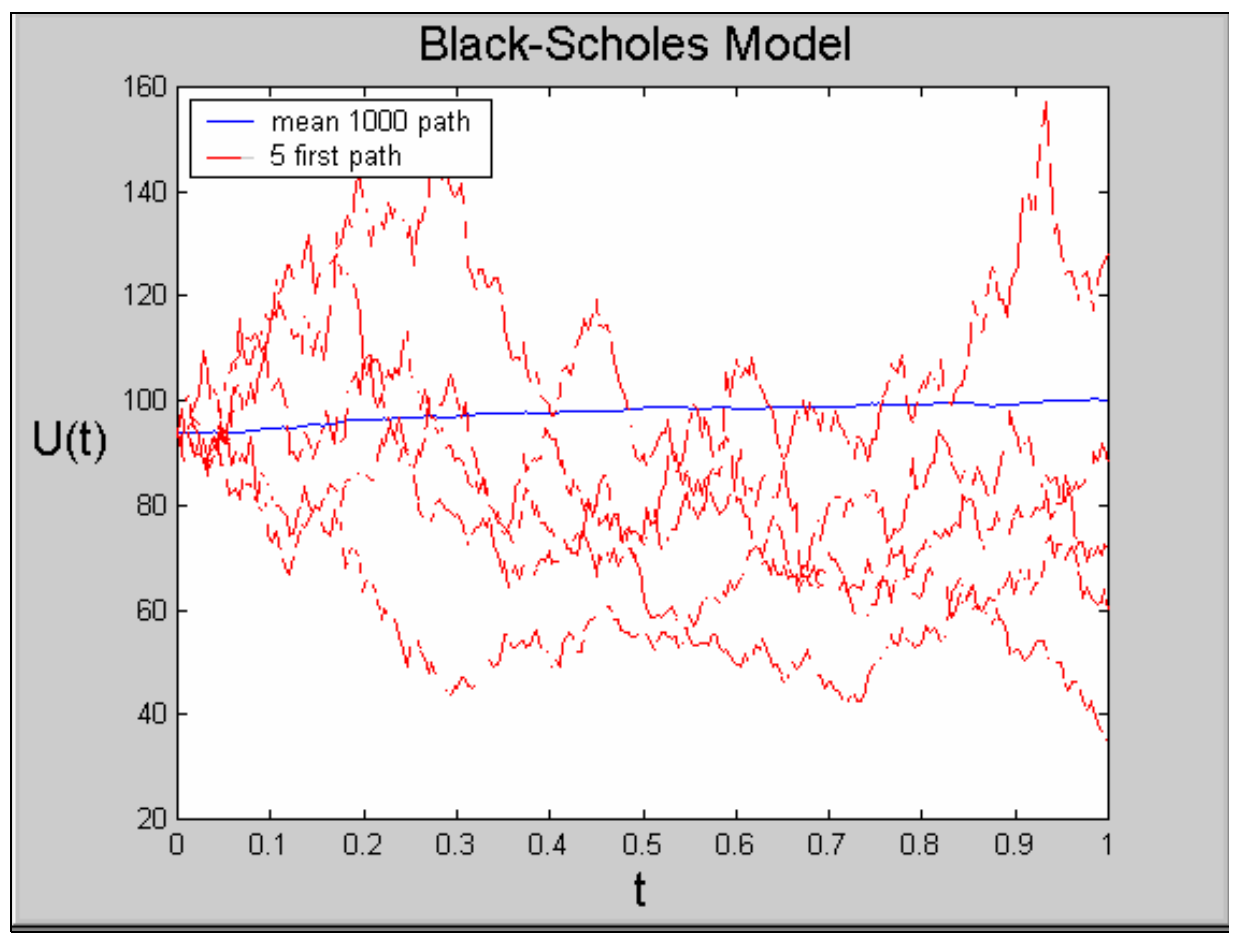

Figura 11. Simulación del modelo BSM

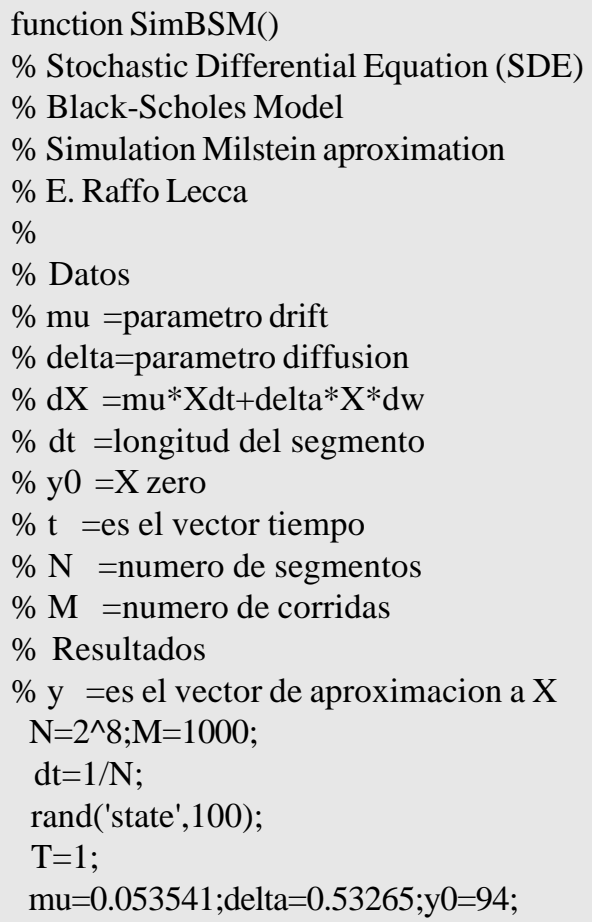

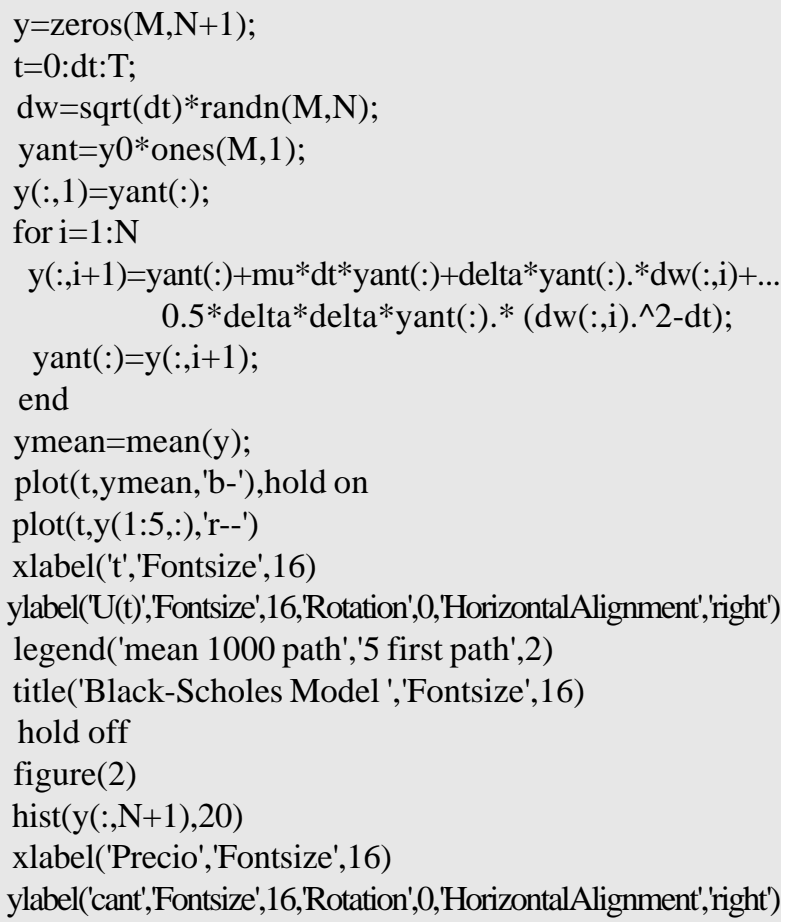




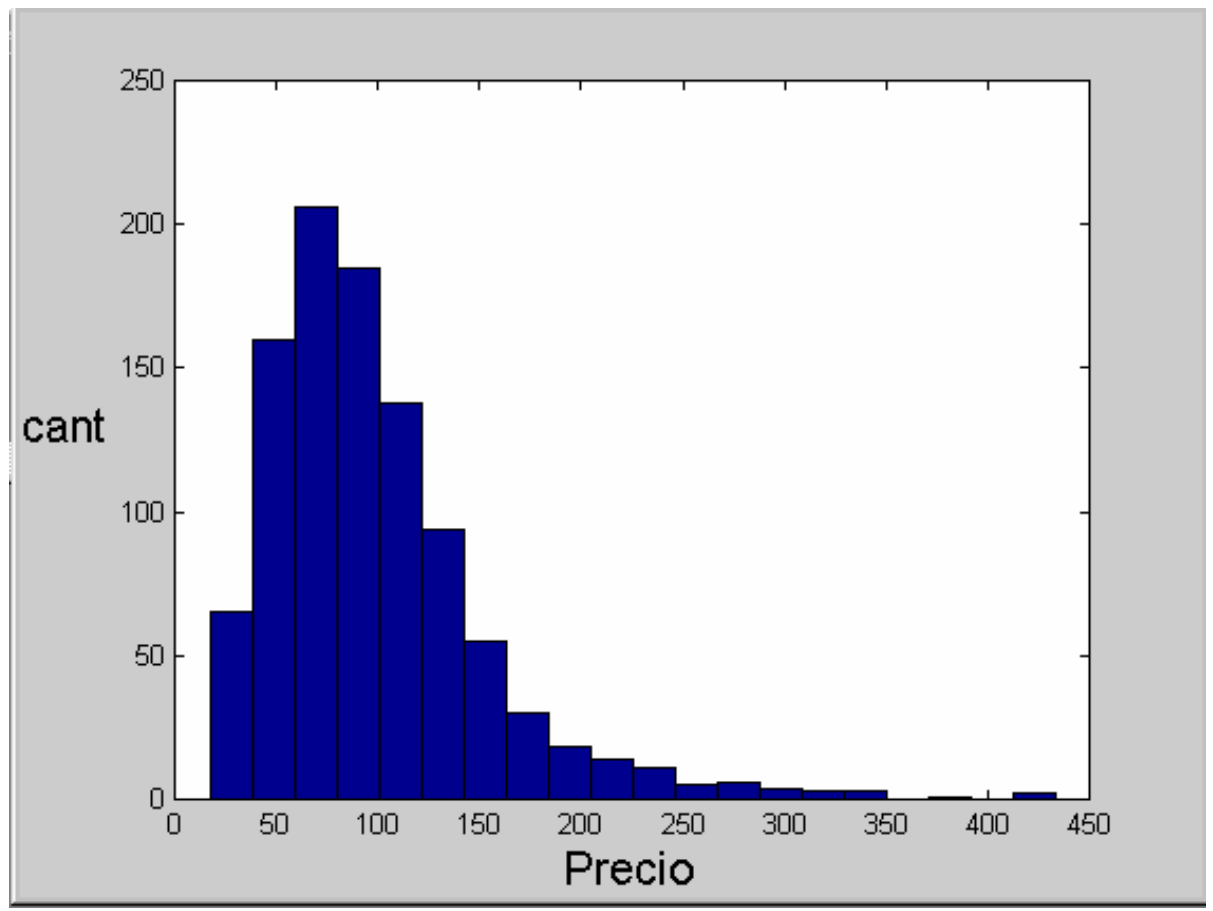

Figura 13. Histograma del precio final

Como se ha podido apreciar en este mundo globalizado, las matemáticas ya son una buena teoría, con gran futuro en las áreas de finanzas de cualquier empresa.

\section{REFERENCIAS BIBLIOGRÁFICAS}

1. Burke, Dave, "An Extensible $\mathrm{C}++$ Framework for Stochastic Differential Equations", $\mathrm{C} / \mathrm{C}++$ Users Journal, November 2003, Volume 21, Number 11.

2. Kloeden, Peter E., Eckhard Platen, (1999) "Numerical Solution of Stochastic Differential Equations", Third Edition, Springer-Verlag.

3. Oksendal, Bernt, (1998), "Stochastic Differential Equations: An Introduction with Applications", 5th Edition, Springer-Verlag.

4. Raffo Lecca, E., (2005) "Métodos Numéricos para Ciencia e Ingeniería con MATLAB" , Lima, Perú.
5. Raffo Lecca, E., (2006) "Programas MATLAB@, para SDE", http://industrial.unmsm.edu.pe/org/ apya/daisi/eraffol/website/index.htm

6. Spiegel, Murray R., (1981) "Applied Differential Equations", Prentice-Hall, Inc., Englewood Cliffs, N.J.

7. Stoer, J., R. Bulirsch, (1996) "Introduction to Numerical Analysis", Springer-Verlag, Second Edition.

8. Wikipedia, La enciclopedia libre, http:// es.wikipedia.org/wiki/Opciones_financieras Compra_de_una_Opci.C3.B3n_Call

9. Wilmott, P., (1998), "Derivaties: The Theory and Practices of Financial Engineering", John Wiley \& Sons.

10. Winston, Wayne, (2000), "Financial Models Using Simulation and Optimization", Second Edition, Palisade Corporation. 